\title{
Cerebral Venous Thrombosis due to Nontyphoidal Salmonella Bacteremia
}

\author{
Kenta Taneda ${ }^{1,2}$, Tadashi Adachi ${ }^{1}$, Yasuhiro Watanabe ${ }^{1}$ and Ritsuko Hanajima ${ }^{1}$
}

\begin{abstract}
:
A 19-year-old previously healthy man presented with convulsions, fever, headache, diarrhea, and vomiting. Brain magnetic resonance imaging revealed cerebral hemorrhaging in the right parietal lobe and thrombotic occlusion of the right great cerebral vein. Blood cultures were positive for nontyphoidal Salmonella. The patient was successfully treated with antibiotics and anticoagulants. Nontyphoidal Salmonella bacteremia can cause cerebral venous thrombosis and physicians therefore need to consider nontyphoidal Salmonella bacteremia as a potential cause of cerebral venous thrombosis.
\end{abstract}

Key words: bacteremia, nontyphoidal Salmonella, venous thrombosis, MERS

(Intern Med 58: 1943-1946, 2019)

(DOI: 10.2169/internalmedicine.2266-18)

\section{Introduction}

Cerebral venous thrombosis (CVT) is a rare disorder with a reported incidence of $0.5 \%$ to $1 \%$ of all strokes, but it is potentially life-threatening. The risk factors for CVT include inherited thrombophilia, pregnancy, antiphospholipid syndrome, and cancer. Infection is also a major cause of this disorder (1). Salmonella is a genus of rod-shaped Gramnegative bacteria. Salmonella species are food and waterborne pathogens, and infection results in a variety of presentations, including enteritis, fever, and bacteremia and it may spread to any site in the body, including the brain. We herein report a rare case of CVT due to Salmonella infection.

\section{Case Report}

A 19-year-old previously healthy man was admitted to our hospital with convulsions, fever, headache, diarrhea, and vomiting. He had no pets and had eaten noodles with boiled eggs at a restaurant 7 days before admission. He had developed abdominal pain and diarrhea a couple of days thereafter and had visited a nearby hospital to receive antidiarrheals, after which his abdominal symptoms had improved immediately. Several days later, his mother had found him in a state of tonic seizure and requested an ambulance. His body temperature was $37.2^{\circ} \mathrm{C}$. Physical examination revealed convulsions, headache, diarrhea, and vomiting, with increased bowel sounds. The cardiovascular and respiratory findings were unremarkable, meningeal signs were absent, and no Osler's node and Janeway lesions were observed. No neurological abnormalities other than the seizure were found. An initial hematological investigation revealed a white blood cell count of $13.7 \times 10^{3} / \mu \mathrm{L}$, with $86 \%$ neutrophils with no renal dysfunction or hypocomplementemia.

The D-dimer level was slightly elevated $(3.3 \mu \mathrm{g} / \mathrm{mL})$, but antithrombin III was $90 \%$, protein C activity was $144 \%$ and protein $\mathrm{S}$ activity was $101 \%$, so there was no evidence of inherited thrombophilia, and anti-cardiolipin antibody was not elevated. Two sets of blood cultures performed at the same time were positive for Salmonella spp. with $\mathrm{O} 4$ and Ha antigens. Brain computed tomography (CT) and magnetic resonance imaging revealed spotty cerebral hemorrhaging in the right parietal lobe and thrombosis of the right parietal cortical vein (Fig. 1). Magnetic resonance venography showed no occlusion of the superior sagital sinus, right transverse sinus, and right sigmoid sinus (Fig. 2A). A transient high-intensity area was seen in the splenium of the corpus callosum on diffusion-weighted images (Fig. 2B), so-

${ }^{1}$ Division of Neurology, Department of Brain and Neurosciences, Faculty of Medicine, Tottori University, Japan and ${ }^{2}$ Department of Neurology, Japanese Red Cross Matsue Hospital, Japan

Received: October 15, 2018; Accepted: January 7, 2019; Advance Publication by J-STAGE: February 25, 2019

Correspondence to Dr. Tadashi Adachi, adachi.neuro@gmail.com 

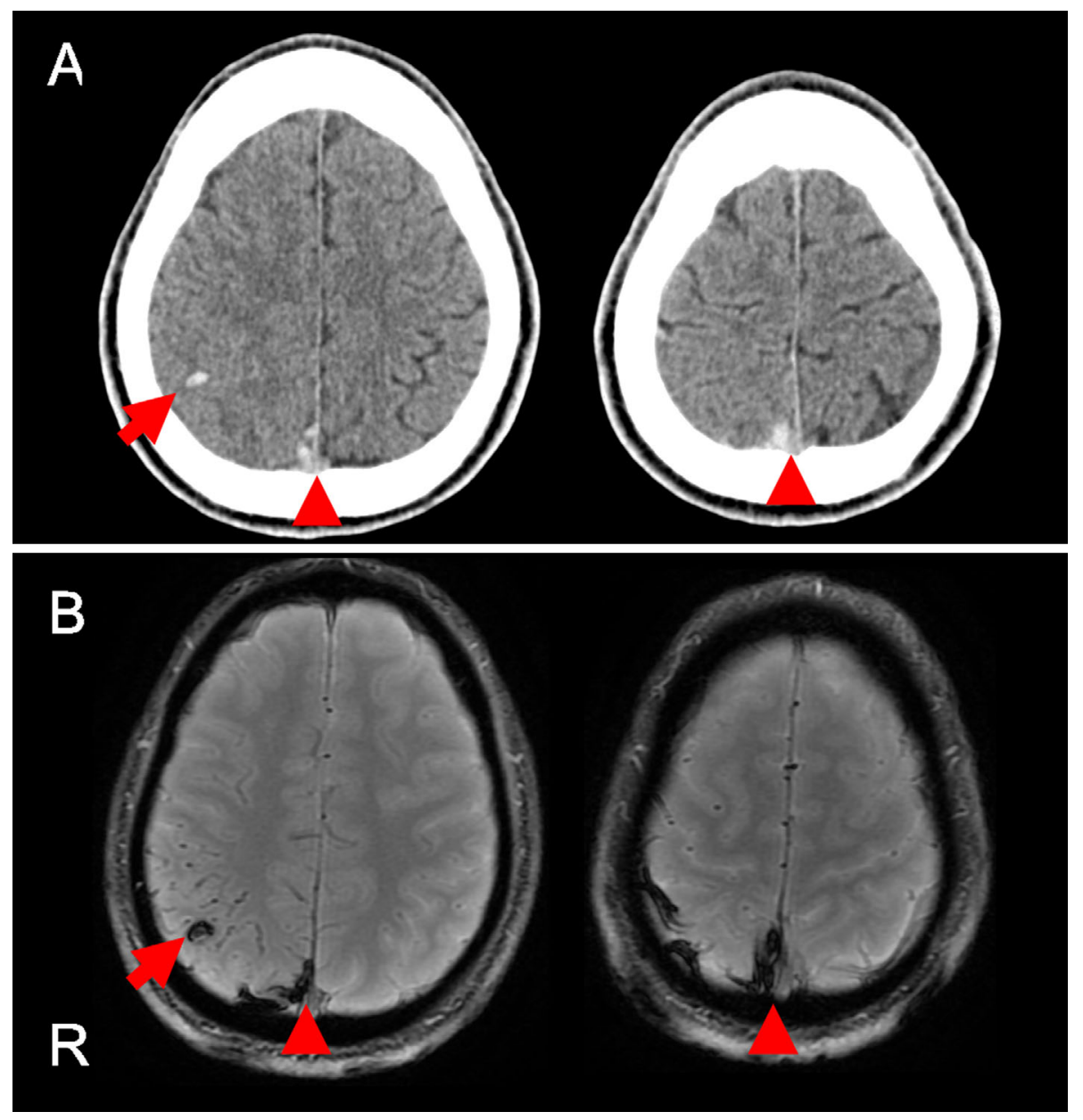

Figure 1. Brain computed tomography (CT) and magnetic resonance imaging (MRI) findings in our patient. Brain CT (A) and MRI T2* weighted images (B) showed right parietal hemorrhaging (arrows) and a thrombus in the suprasagittal sinus (arrowheads).
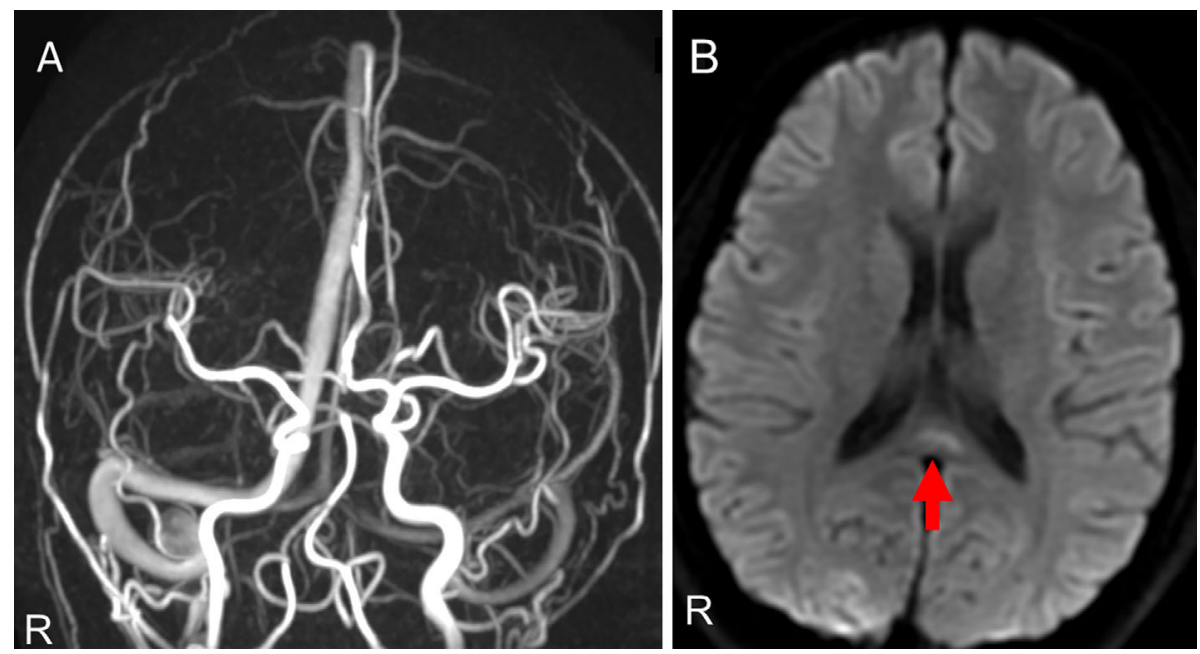

Figure 2. Magnetic resonance (MR) venography and diffusion-weighted imaging (DWI) findings. MR venography showed no occlusion of the major cerebral veins (A). The splenium of the corpus callosum demonstrated hyperintensity on DWI (B; arrow).

called clinically mild encephalitis/encephalopathy with a reversible splenial lesion (MERS) (2). Electroencephalography was normal. No other focus of infection was detected on systemic CT scans.
Tonic clonic convulsion was successfully treated by intravenous diazepam, and fosphenytoin was administered to prevent a recurrence of the seizure. Anticoagulant therapy was initiated with heparin 10,000 units/day, and warfarin was 
gradually substituted for the heparin. Empiric antimicrobial therapy with intravenous ceftriaxone ( 2 g every 24 hours) was started for the Salmonella bacteremia. Ceftriaxone was used for four days, and then it was replaced by intravenous levofloxacin for twelve days because of an elevation of liver enzymes. Stool cultures after the end of antibiotic administration were positive for Candida glabrata, but Salmonella was not detected, and blood cultures were negative after antibiotic therapy. Twenty-two days after admission, the patient was discharged with no neurological impairment. He was thereafter administered warfarin and levetiracetam for three months and had no recurrence of thrombosis or convulsion. Moreover, the thrombosis of the right great cerebral vein was found to have disappeared.

\section{Discussion}

This case is unique in two aspects. First, it is rare for nontyphoidal Salmonella bacteremia to occur in a previously healthy subject. Salmonella is a genus of rod-shaped Gramnegative bacteria. Salmonella species can be found in the gastrointestinal tracts of humans and animals-especially reptiles and birds. Infection is spread by contaminated food and water, and the incubation period varies from 4 to 72 hours. Salmonella serotypes can be divided into typhoidal and nontyphoidal. Nontyphoidal serotypes are the most common and cause self-limiting gastrointestinal disease, but bacteremia occurs in $5 \%$ to $10 \%$ of all infected persons (3). The risk of bloodstream infection depends on the host's immune system and the degree of bacterial virulence; infancy, immune deficiency, malnutrition, malaria, and anemia are well-known risk factors (4-6). Most adults who develop nontyphoidal Salmonella bacteremia have predisposing diseases and are usually already receiving medications, particularly immunosuppressive agents (6). Some types of salmonella that can easily enter the blood have been identified and include serotype Heidelberg (7). Our patient was a 19-year-old male with no medical history of note, but the use of antidiarrheals may have prolonged the infection or led to the onset of bacteremia (8).

Second, it is rare that nontyphoidal Salmonella bacteremia caused CVT. Usually, this bacteria often affects young patients and can cause variable neurological symptoms, such as headache, papilledema, diplopia, and other focal neurological deficits. These clinical symptoms are related to increased intracranial pressure and focal brain injury. However, in our case, there was no focal injury and MRI showed MERS, so the clinical symptoms of our case are therefore considered to have been caused by CVT. Inherited thrombophilia, pregnancy, antiphospholipid syndrome, cancer, and other uncommon causes are associated with CVT (9). Parameningeal infection has predominated in previous cases of infectious CVT (1), and there are only a limited number of reported cases of CVT due to Salmonella bacteremia (10-12). Initial treatment with anticoagulants is effective for preventing further thrombus development and facilitating recanalization. In this case, no underlying cause of CVT except for the Salmonella bacteremia was detected. In a mouse model, systemic Salmonella typhimurium infection was reported to induce thrombosis via the ligation of C-type lectin-like receptor-2 on platelets (13), and a similar mechanism likely operates in humans. Moreover, adults with nontyphoidal Salmonella bacteremia are at risk for developing infected aortic aneurysms (14). Taken together, these results suggest that nontyphoidal Salmonella has a high affinity for the vascular system. Our patient was successfully treated with appropriate antibiotic and anticoagulant therapy. Both CVT and Salmonella bacteremia are uncommon, but lifethreating conditions. Clinicians should therefore consider Salmonella bacteremia as a potential cause of CVT.

The authors state that they have no Conflict of Interest (COI).

\section{References}

1. Saposnik G, Barinagarrementeria F, Brown RD Jr, et al. Diagnosis and management of cerebral venous thrombosis: a statement for healthcare professionals from the American Heart Association/ American Stroke Association. Stroke 42: 1158-1192, 2011.

2. Takanashi J. Two newly proposed infectious encephalitis/encephalopathy syndromes. Brain Dev 31: 521-528, 2009.

3. Hammack T. Salmonella species. In: Bad Bug Book e Handbook of Foodborne Pathogenic Microorganisms and Natural Toxins. 2nd ed. Lampel KA, Ed. Food and Drug Administration, Washington DC, 2012: 12-16.

4. Takem EN, Roca A, Cunnington A. The association between malaria and non-typhoid Salmonella bacteraemia in children in subSaharan Africa: a literature review. Malar J 13: 400, 2014.

5. Gordon MA. Salmonella infections in immunocompromised adults. J Infect 56: 413, 2008.

6. Shimoni Z, Pitlik S, Leibovici L, et al. Nontyphoid Salmonella bacteremia: age-related differences in clinical presentation, bacteriology, and outcome. Clin Infect Dis 28: 822-827, 1999.

7. Asmar BI, Abdel-Haq N. Nontyphoidal Salmonella infection in children: relation to bacteremia, age, and infecting serotype. Infect Dis 48: 147-151, 2016.

8. DuPont HL, Hornick RB. Adverse effect of lomotil therapy in shigellosis. JAMA 226: 1525-1528, 1973.

9. Ferro JM, Canhão P, Stam J, Bousser MG, Barinagarrementeria F; ISCVT Investigators. Prognosis of cerebral vein and dural sinus thrombosis results of the International Study on Cerebral Vein and Dural Sinus Thrombosis (ISCVT). Stroke 35: 664-670, 2004.

10. Pineda MC, Lopinto-Khoury C. Cerebral venous sinus thrombosis secondary to typhoid fever: a case report and brief summary of the literature. Neurologist 18: 202-203, 2012.

11. Okunola PO, Ofovwe GE, Abiodun MT, Azunna CP. Superior sagittal sinus thrombosis complicating typhoid fever in a teenager. Case Reports in Pediatrics Volume 2012: 2012.

12. Inghilleri M, Pedace F, Argenta $M$, et al. Thrombosis of cerebral veins dural sinuses after paratyphi. Ital J Neurol Sci 16: 257-259, 1995.

13. Jessica RH, Charlotte NC, Saeeda B, et al. Inflammation drives thrombosis after Salmonella infection via CLEC-2 on platelets. J Clin Invest 125: 4429-4446, 2015.

14. Hsu RB, Chen RJ, Wang SS, Chu SH. Infected aortic aneurysms: clinical outcome and risk factor analysis. J Vasc Surg 40: 30-35, 2004.

The Internal Medicine is an Open Access journal distributed under the Creative 
Commons Attribution-NonCommercial-NoDerivatives 4.0 International License. To view the details of this license, please visit (https://creativecommons.org/licenses/ by-nc-nd/4.0/)

(C) 2019 The Japanese Society of Internal Medicine Intern Med 58: 1943-1946, 2019 